\title{
Neuroimaging Appearance of Cerebral Malignant Epithelioid Glioneuronal Tumors in Children
}

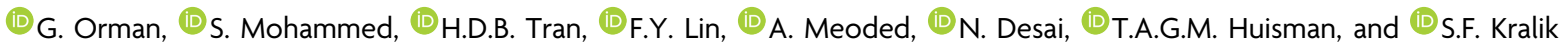

\begin{abstract}
SUMMARY: Malignant epithelioid glioneuronal tumor is a rare high-grade, aggressive brain tumor that shows both glial and neuronal differentiation on histopathology but is not included in the current World Health Organization classification. The neuroimaging appearance is variable but may be secondary to the size of the mass and/or location of the tumor. In our series, all epithelioid glioneuronal tumors were encountered in the supratentorial space and included pineal, temporal, and extratemporal lobar cerebral hemisphere locations. When large, the tumors demonstrate cystic degeneration and necrosis, hemorrhage, contrast enhancement, and regions of low apparent diffusion coefficient scalars consistent with patterns seen with other high-grade pediatric brain tumors. The tumors also have a propensity to spread into the meninges at presentation and for distant CSF spread on follow-up imaging.
\end{abstract}

ABBREVIATION: MEGNT = malignant epithelioid glioneuronal tumor

B rain tumors are the second leading cause of cancer after leukemia in the pediatric population. Pediatric brain tumors are the most common among all causes of death from solid tumors in children. ${ }^{1,2}$ Primary brain tumors are more common and are of various histologic types. Their prognosis and treatment depend on the histologic type, tumor grade, and, increasingly, the molecular features. ${ }^{2}$

Malignant epithelioid glioneuronal tumor (MEGNT) is a rare high-grade, aggressive brain tumor that shows both glial and neuronal differentiation on histopathology. MEGNT is not included in the current World Health Organization classification. ${ }^{3}$ Patients usually present with signs and symptoms of increased intracranial pressure or symptoms related to the location of the lesion. Both pediatric and adult cases have been described in the neuropathology literature. ${ }^{4-7}$ The first report described clinicopathologic features of 2 unusual MEGNTs in pediatric patients. The tumors shared histopathology and clinical aggressiveness with 3 cases previously described in adults. ${ }^{5,7}$ The largest clinicopathologic study included 40 patients; 15\% (6/40) of the patients were children. The authors evaluated all tumors diagnosed as MEGNTs in routine practice during a 2-year period during which

Received February 14, 2020; accepted after revision May 28.

From the Edward B. Singleton Department of Radiology (G.O., S.M., H.D.B.T., A.M., N.D., T.A.G.M.H., S.F.K.), and Department of Oncology (F.Y.L.), Texas Children's Hospital and Baylor College of Medicine, Houston, Texas.

Please address correspondence to Gunes Orman, MD, Texas Children's Hospital, Edward B. Singleton Department of Radiology, 6701 Fannin St, Suite 470, Houston, TX 77030; e-mail: gxorman@texaschildrens.org

http://dx.doi.org/10.3174/ajnr.A6668 neurofilament protein immunostaining was performed in any case of suspected malignant glioma with unusual clinical, radiographic, and/or histologic features. ${ }^{6}$ Neuroimaging is essential for the initial diagnosis; however, a systematic review of the neuroimaging findings of pediatric MEGNT has not been described. ${ }^{4}$ The purpose of this article was to describe the CT and MR imaging features of this rare and aggressive tumor in a pediatric case series.

\section{Case Series}

Following institutional review board approval, a retrospective review of CTs and MRIs from 10 pediatric patients (18 years of age or younger) with a pathology-proved MEGNT diagnosis was performed by 2 board-certified neuroradiologists (N.D., S.F.K.), each with 9 years of neuroradiology experience. Initial-presentation CTs were reviewed to determine the density of the solid portion of the tumor relative to the normal brain parenchyma (hyperdense, isodense, hypodense), the presence of calcification and/or hemorrhage, and bone changes (scalloping, bone erosion). Initial-presentation MRIs were reviewed to determine the following: tumor location; maximum diameter; T2WI appearance relative to the normal brain parenchyma (hyperintense, isointense, hypointense); presence of cysts/necrosis (yes/no); presence of flow voids (yes/no) and perilesional T2WI hyperintensity suggestive of vasogenic edema (none, mild, extensive); minimum ADC value of the solid portion of the tumor using a $5-\mathrm{mm}^{3} \mathrm{ROI}$; presence of hemorrhage or calcification on gradient-echo or specific 
calcification versus hemorrhage determined from phase-contrast images in susceptibility-weighted imaging; enhancement on postcontrast T1-weighted imaging (yes/no); and leptomeningeal/ dural extension (yes/no) determined from postcontrast T1weighted imaging. Initial presentation and follow-up MRIs of the brain and spine were reviewed for presence of CSF metastases in the brain and spine, defined as nodular or linear areas of abnormal enhancement along the meningeal surfaces.

The mean age of the children was 8.7 years (range, 3.818.4 years), and $50 \%$ were female. All tumors were supratentorial in the pineal $(n=2)$, temporal $(n=2)$, and extratemporal cerebral hemispheres including the frontal lobe $(n=3)$ and frontal and parietal lobes $(n=3)$. Of the 8 tumors involving the cerebral hemispheres, 7 tumors (88\%) involved the cortical and subcortical regions, indicative of a peripheral predominance. One tumor involving the frontal and parietal lobes demonstrated 2 separate enhancing masses and was classified as multifocal, while the remainder demonstrated 1 single enhancing mass. Presenting signs and symptoms included headache, vomiting, abnormal gait, seizure, lethargy, hemiparesis, and confusion. All tumors underwent surgical resection followed by variable treatment, including chemotherapy and/or radiation therapy. Three patients died of the complications of their brain tumors.

The CT appearance of MEGNT was isodense (80\%) and hyperdense (20\%). Other CT findings included acute hemorrhage (10\%), calcification (10\%), bone scalloping (10\%), and bone erosion (10\%).

The MR imaging features of MEGNT were variable as seen in the Table. As a group, MEGNTs presented with a mean tumor diameter of $5.3 \mathrm{~cm}$ (range, $2.5-7.7 \mathrm{~cm}$ ) and appeared T2WI isointense (50\%) and hyperintense (40\%), cystic-like and/or necrotic (80\%) with T2WI flow voids (60\%), perilesional vasogenic edema (none $20 \%$, mild $30 \%$, extensive $50 \%$ ), calcification/hemorrhage (50\%), enhancement (100\%), leptomeningeal/dural extension $(50 \%)$, and an average minimum ADC value of $737 \times 10^{-6} \mathrm{~mm}^{2} / \mathrm{s}$ (range, $560-1158 \times 10^{-6} \mathrm{~mm}^{2} / \mathrm{s}$ ).

When we considered MEGNT by location of pineal, temporal, or extemporal lobar tumors, a more noticeable pattern was evident as seen in the Table. Smaller means and ranges of maximum

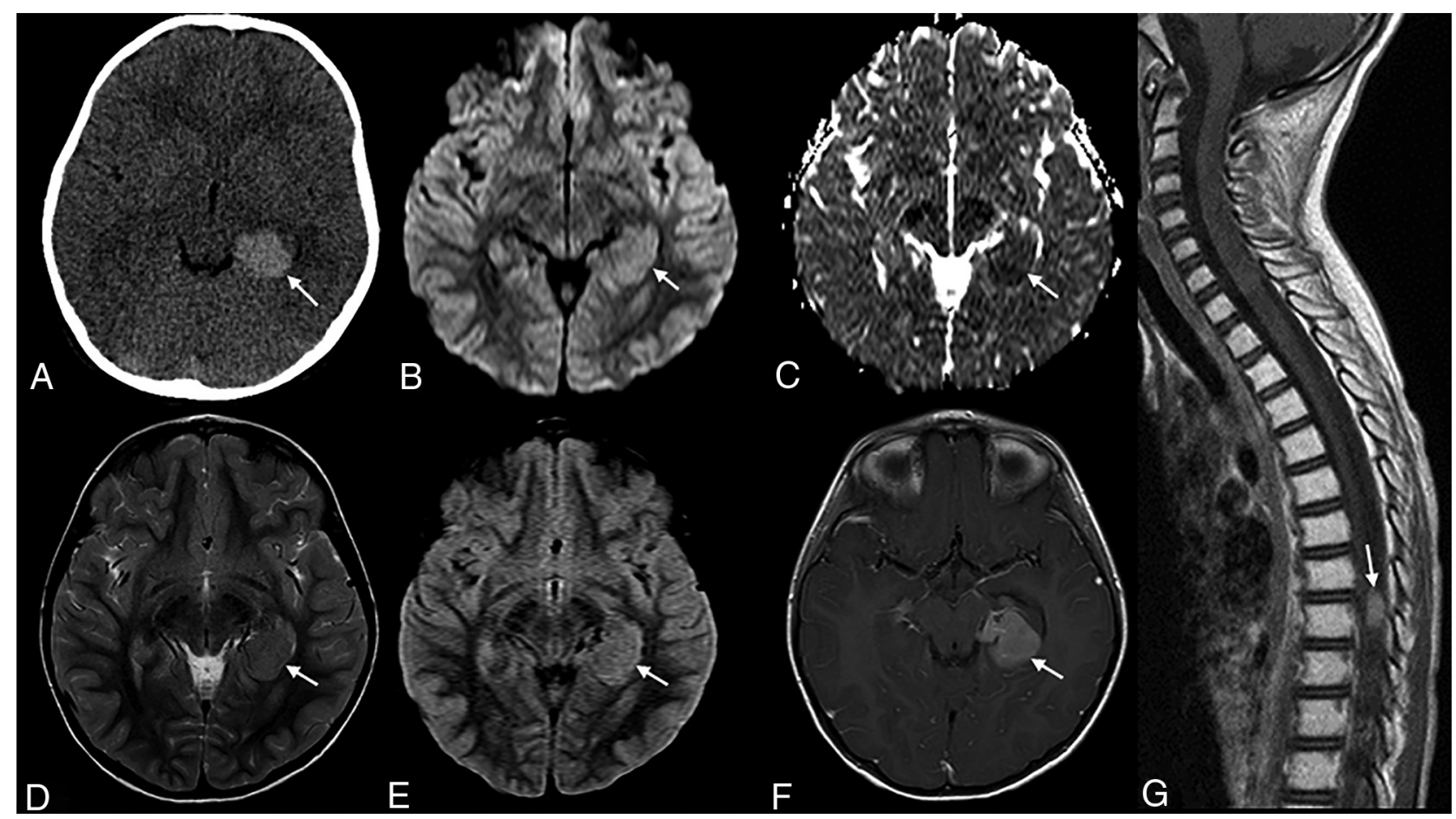

FIG 1. A 5-year-old girl with morning vomiting and headaches. $A$, Axial non-contrast-enhanced $C T$ shows an oval homogeneous hyperdense mass lesion (arrow) located along the mesial left temporal lobe. $B$ and $C$, Diffusion-weighted imaging and apparent diffusion coefficient map show no restricted diffusion (arrow) of the lesion. $D$ and $E$, The lesion is nearly isointense (arrow) to gray matter on the T2-weighted and FLAIR imaging (F). The lesion shows avid homogeneous enhancement (arrow) on postcontrast T1-weighted imaging. The adjacent brain shows no edema. G, Followup sagittal postcontrast T1-weighted spine imaging after 6 months shows drop metastasis (arrow) with avid homogeneous enhancement. 


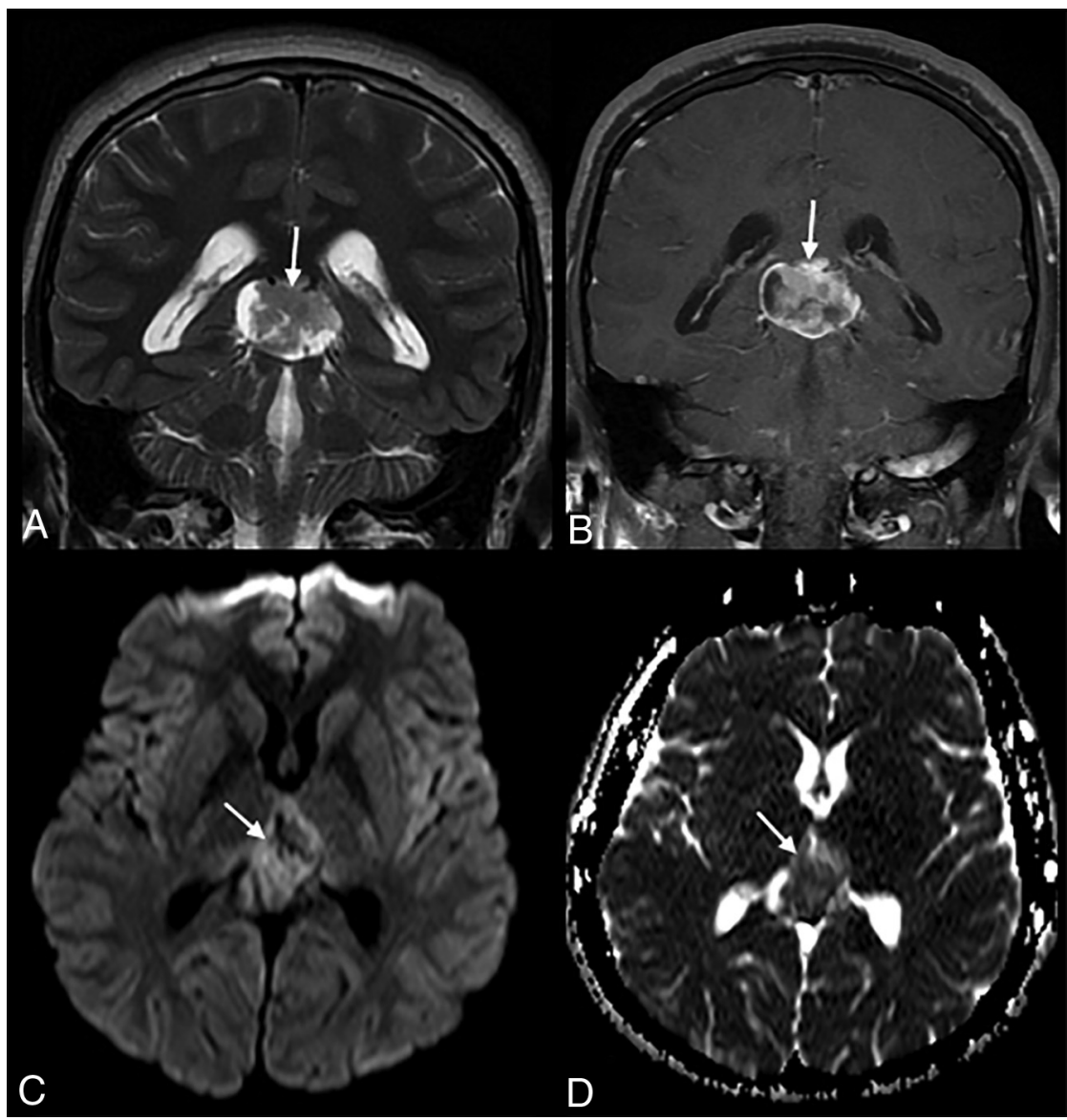

FIG 2. An 18-year-old man with a recent presentation of a 2-day history of headache and vomiting. A, Coronal T2-weighted imaging shows a large mass lesion (arrow) in the pineal gland region. $B$, The lesion shows heterogeneous contrast enhancement (arrow) on coronal T1-weighted imaging, with mild restricted diffusion (arrow, $C$ and $D$ ) on diffusion-weighted imaging and the matching apparent diffusion coefficient map.

\section{DISCUSSION}

MEGNT is a rare aggressive brain tumor that can be encountered in both children and adults. ${ }^{4,7}$ Neuroimaging features of MEGNT were described in only a few articles in the literature with various CT and MR imaging findings. ${ }^{4,6}$ Varlet et $\mathrm{al}^{6}$ reported a series of 40 cases, including both adults and children, of all tumors diagnosed as MEGNT in routine practice during a 2-year period, during which neurofilament protein immunostaining was performed in any case of suspected malignant glioma with unusual clinical, radiographic, and/or histologic features. Preoperative CT or MR imaging or both were reviewed in all except 1 case. Only $15 \%$ (6/40) of patients experienced initial symptoms during childhood (age range, $7-11$ years). ${ }^{6}$ To the best of our knowledge, our article is the largest case series focusing on neuroimaging features of pediatric MEGNT.

All tumors were supratentorial in our series, similar to the previously reported cases, being nearly always supratentorial, and most were in a temporal or frontal location. ${ }^{4,6}$ Different from Varlet et al, ${ }^{6}$ we encountered pineal region MEGNTs in 2 patients, which may indicate a more unique location in children compared with adults.

In our study, most cases $(80 \%)$ tumor diameters were seen with temporal tumors $(3.2 \mathrm{~cm}$; range, $2.9-3.4 \mathrm{~cm})$ and pineal tumors $(2.7 \mathrm{~cm}$; range, $2.5-2.8 \mathrm{~cm})$ compared with extratemporal tumors $(6.8 \mathrm{~cm}$; range, $5.4-7.8 \mathrm{~cm})$. Cysts or necrosis or both were seen in all pineal (100\%) and extratemporal tumors (100\%) but in none of the temporal tumors (0\%). Leptomeningeal/dural extension was more frequent with extratemporal tumors $(100 \%)$ but was not seen with temporal $(0 \%)$ or pineal (0\%) tumors. Perilesional edema was present more frequently with extratemporal $(100 \%)$ tumors than with temporal $(50 \%)$ or pineal (50\%) tumors. Flow voids were more frequent in extratemporal tumors $(83 \%)$ compared with temporal $(50 \%)$ and pineal tumors (0\%). Calcification or blood products were similar for temporal (50\%), pineal (50\%), and extratemporal (50\%) tumors. Mean and ranges of $\mathrm{ADC}$ values were overlapping for temporal $(795 \times$ $10^{-6} \mathrm{~mm}^{2} / \mathrm{s}$; range, $\left.764-806 \times 10^{-6} \mathrm{~mm}^{2} / \mathrm{s}\right)$, pineal $(1008 \times$ $10^{-6} \mathrm{~mm}^{2} / \mathrm{s}$; range, $\left.858-1158 \times 10^{-6} \mathrm{~mm}^{2} / \mathrm{s}\right)$, and extratemporal tumors $\left(658 \times 10^{-6} \mathrm{~mm}^{2} /\right.$; range, $\left.560-899 \times 10^{-6} \mathrm{~mm}^{2} / \mathrm{s}\right)$.

Although no patient had remote CSF metastases at initial presentation, 3 patients (30\%) developed distant leptomeningeal metastasis on follow-up imaging within both the brain and spine from 3 to 5 months from initial presentation. Representative examples of CT and MR imaging patterns of MEGNT are seen in Figs 1-5. were isodense on CT, with the uncommon presence of hemorrhage $(10 \%)$ or calcification (10\%). Similarly, calcifications on CT were reported in $17.6 \%$ of cases by Varlet et al. ${ }^{6}$ An unusual finding on $\mathrm{CT}$ in one of our patients was an associated calvarial bone erosion and bone scalloping adjacent to the brain tumor, which have not been previously reported with MEGNT, likely because most of the nonpineal tumors involved the cortical and subcortical areas (88\%) and leptomeningeal/dural extension was frequently identified on imaging and in pathology descriptions. This propensity for cortical involvement and leptomeningeal/ dural extension is one feature that may distinguish this tumor type from other high-grade pediatric brain tumors and was not systematically reported in the literature.

Necrotic and/or cystic-like components were present in $80 \%$ of our patients, similarly reported in $85 \%$ of previous published series. We found contrast enhancement on MR imaging in $100 \%$ of patients, similar to the description of marked contrast enhancement on neuroimaging reported in $97.5 \%$ of patients in a prior study. ${ }^{6}$ As demonstrated in our subgroup analysis of MEGNT by location and size, MEGNTs arising from the extratemporal cerebral hemispheres appear to be larger and more heterogeneous in appearance than the smaller MEGNTs in the 


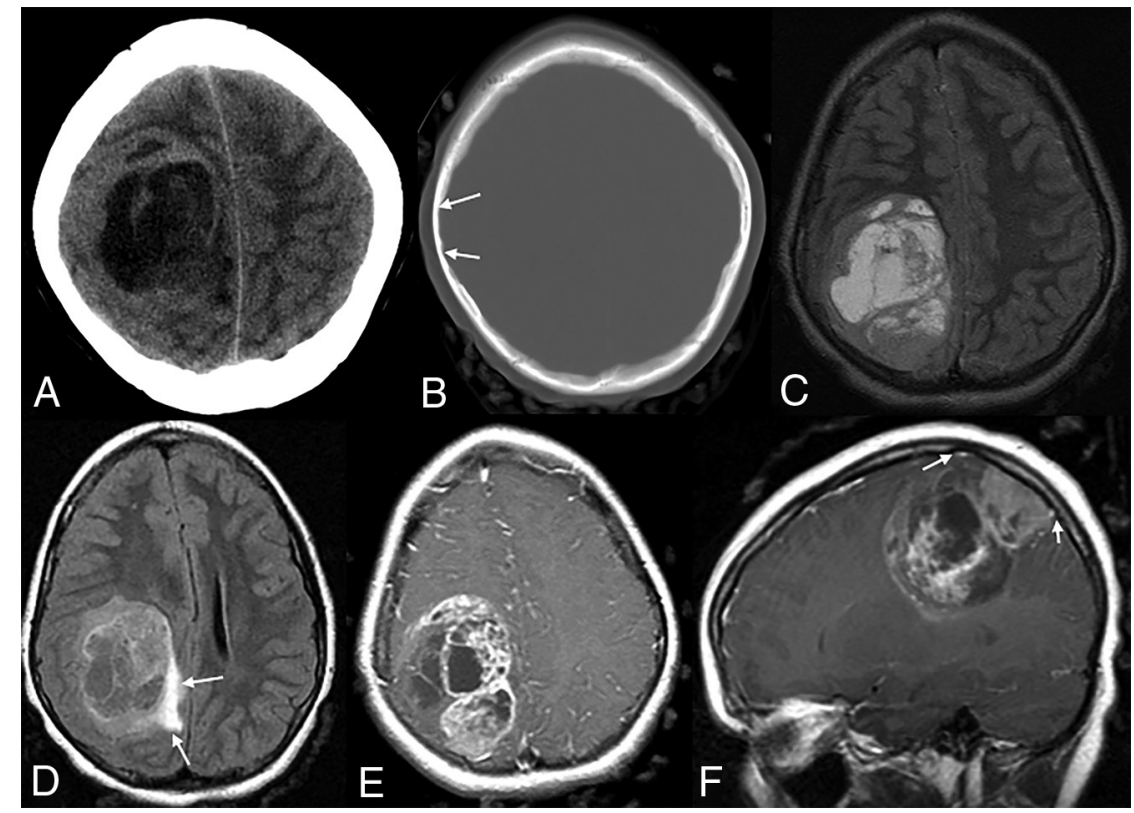

FIG 3. An 8-year-old girl with no medical history available in the patient records. $A$ and $B$, Axial $C T$ images demonstrate a solid and cystic mass with solid portions isodense to normal brain parenchyma and scalloping ( $B$, arrows) of the adjacent inner table of the parietal bone. $C-F, M R$ images demonstrate a large right frontoparietal mass with extensive cystic/necrotic areas $(C)$, surrounding edema (arrows, $D$ ), enhancement $(E)$, and dural extension (arrows, $F$ ). The lesion causes mass effect and mild right-to-left midline shift. pineal region and temporal lobe. We suspect that as the tumors enlarge, they outgrow their blood supply and become more necrotic, thus accounting for the difference in imaging appearance. The patients with pineal tumors in our series presented with hydrocephalus, and the patient with one of the temporal tumors presented with a new onset of seizure, which may explain their smaller size at presentation. This subgroup analysis was chosen on the basis of prior reports of temporal lobe predilection of MEGNT and the notable differences encountered in this study. ${ }^{6}$ The small sample size of our study limits the ability to correlate patient age and imaging features or tumor size.

Because prior studies have demonstrated that ADC values correlate with low-grade and high-grade tumors in pediatric patients with brain tumors, we found a similar pattern of low ADC values in MEGNT, consistent with a high-grade tumor. ${ }^{8}$ Although ADC values for MEGNT have not

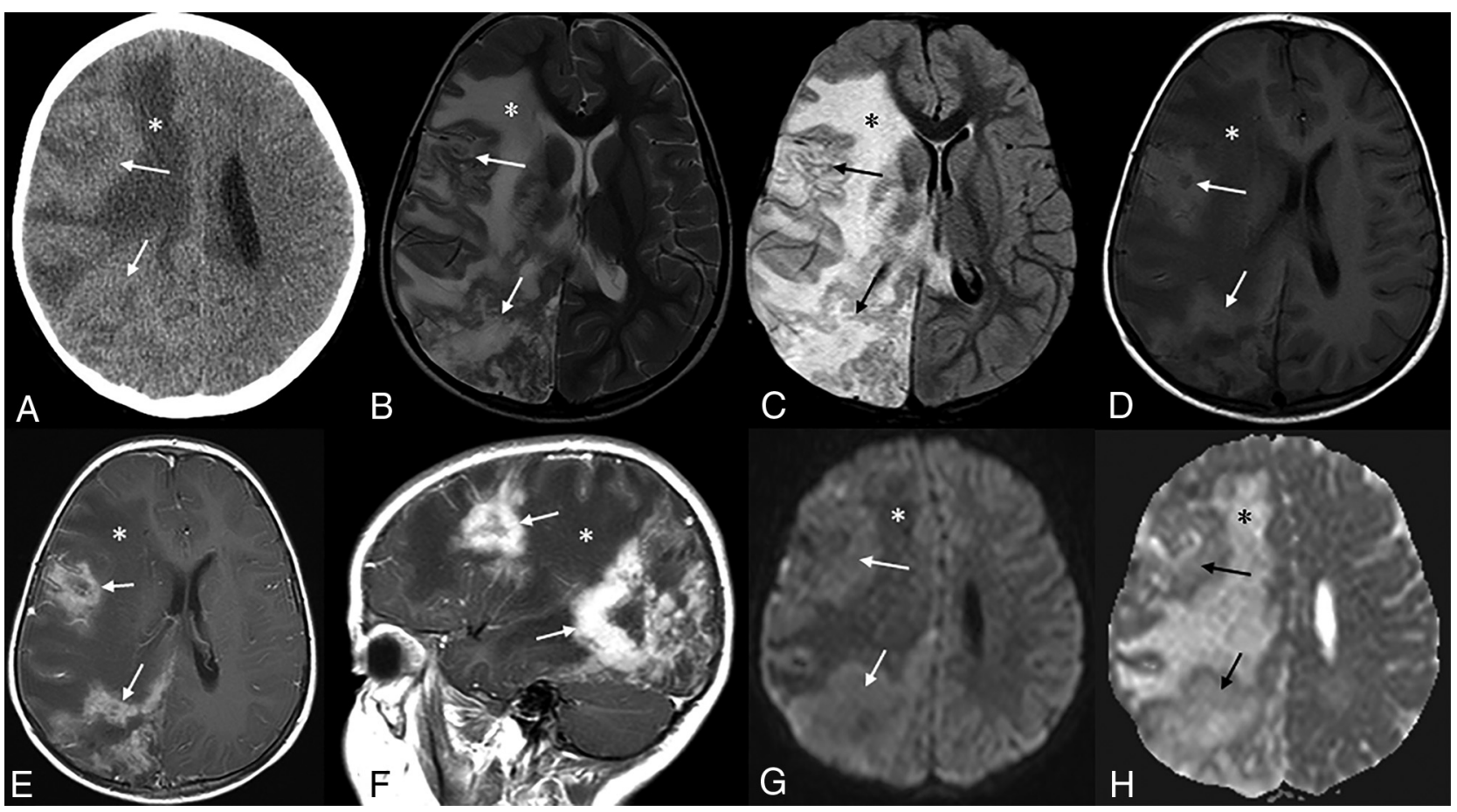

FIG 4. A 3-year-old girl who presented with left-sided hemiparesis. A, Axial noncontrast CT image demonstrates multifocal masses (arrows) in the right cerebral hemisphere that are isodense to normal brain and associated with a large region of edema (asterisk). $B-H, M R$ images demonstrate an infiltrative right cerebral hemisphere tumor (arrows) extending into the basal ganglia and thalamus, with subsequent mass effect, diffuse right cerebral hemispheric edema (asterisk), and right-to-left midline shift. Multifocal areas of contrast enhancement (arrows) are seen on axial and sagittal T1 weighted imaging with contrast ( $E$ and $F$ ). DWI and ADC map images demonstrate that the solid portion of the mass (arrows) is isointense to normal brain parenchyma $(G$ and $H)$. 


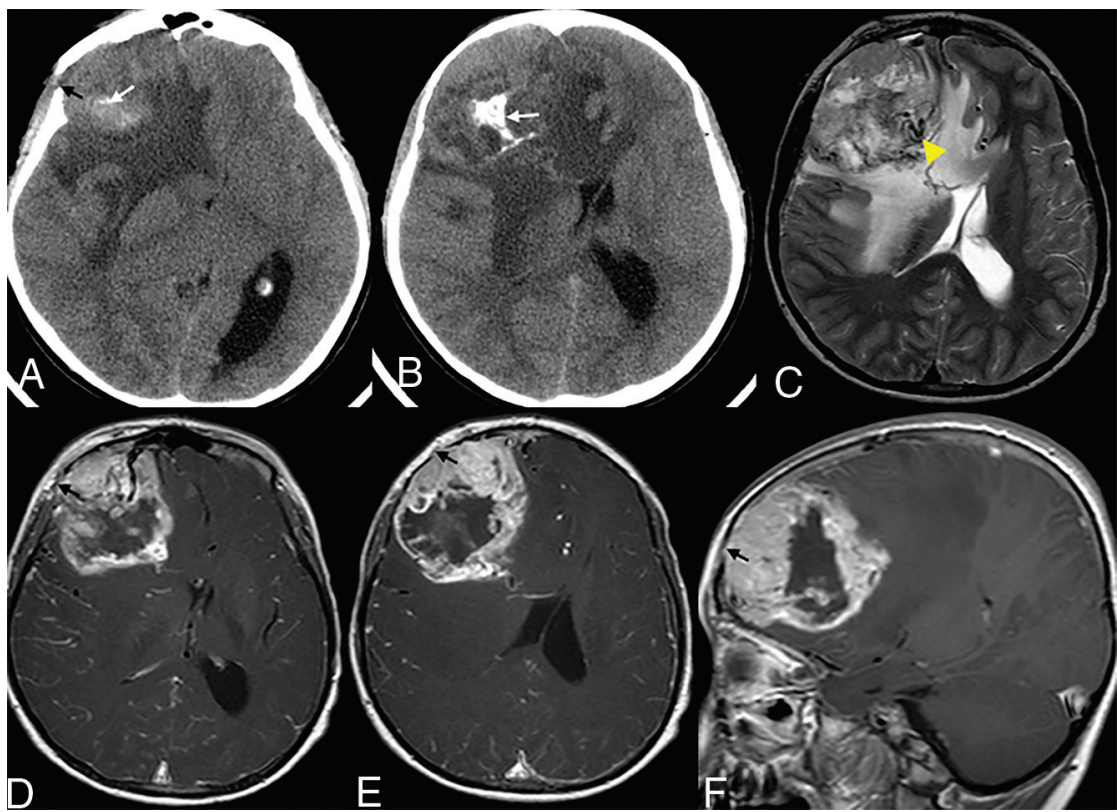

FIG 5. A 5-year-old boy who presented with vision loss. A and B. Axial noncontrast CT images demonstrate a solid and cystic mass with dystrophic calcification (white arrow) and adjacent bone erosion (black arrow). C-F, MR images demonstrate a right frontal lobe tumor with cystic/ necrotic areas, flow voids ( $C$, arrowhead), surrounding edema, contrast enhancement of the tumor, dural extension, and focal areas of bone erosion ( $D-F$, black arrow). There is notable intracranial mass effect, including a right-to-left midline shift. propensity for distant CSF spread at a rate similar to that of medulloblastoma so that routine imaging surveillance of the spine is suggested.

\section{CONCLUSIONS}

MEGNT is a rare and aggressive tumor that can be seen in both the pediatric and adult populations. Neuroimaging features are not specific for a diagnosis, but the presence of cystic degeneration/necrosis, hemorrhage, contrast enhancement, and regions of low ADC signal of the tumors is consistent with patterns seen with other high-grade pineal and cerebral pediatric brain tumors.

\section{REFERENCES}

1. Poussaint TY, Panigrahy A, Huisman TA. Pediatric brain tumors. Pediatr Radiol 2015;45(Suppl 3):S443-53 CrossRef Medline

2. Koob M, Girard N. Cerebral tumors: specific features in children. Diagn Interv Imaging 2014;95:965-83 CrossRef Medline

been previously reported, the mean ADC value of MEGNTs in this series is similar to that in a previous study that found a mean, minimum ADC value of $640 \times 10^{-6} \mathrm{~mm}^{2} / \mathrm{s}$ for supratentorial high-grade brain tumors in children in the first year of life. ${ }^{9}$

The imaging pattern of cystic degeneration/necrosis, hemorrhage, contrast enhancement, and regions of low ADC values of the tumors in our case series is not pathognomonic of MEGNT but is consistent with patterns seen with other high-grade pediatric brain tumors. ${ }^{3}$ While no imaging feature is pathognomonic of MEGNT, the imaging features encountered with MEGNT support its designation as a high-grade tumor. The imaging patterns of pediatric MEGNT described herein may be clinically useful for radiologists encountering these rare tumors. The differential diagnosis should include glioblastoma multiforme, embryonal CNS tumor, anaplastic ependymoma, hemangiopericytoma, and atypical teratoid/rhabdoid tumor. In the pineal location, pineoblastoma would also be in the differential diagnosis. MEGNTs were found only in the supratentorial location and involved older children, which may indicate a less likely diagnosis of an atypical teratoid/rhabdoid tumor. ${ }^{4}$ Last, MEGNTs demonstrated a
3. Johnson DR, Guerin JB, Giannini C, et al. 2016 updates to the WHO Brain Tumor Classification System: what the radiologist needs to know. Radiographics 2017;37:2164-80 CrossRef Medline

4. Rivera-Zengotita M, Rauch RA, Adesina AM, et al. Malignant epithelioid glioneuronal tumor. In: Adesina AM, Tihan T, Fuller CE, eds. Atlas of Pediatric Brain Tumors. Springer-Verlag; 2016:211-19

5. Rivera-Zengotita $M$, Whitehead W, Chintagumpala $M$, et al. Malignant epithelioid glioneuronal tumor: unusual phenotype or new entity? Mod Pathol 2008;21:324A

6. Varlet P, Soni D, Miquel C, et al. New variants of malignant glioneuronal tumors: a clinicopathological study of $\mathbf{4 0}$ cases. Neurosurgery 2004;55:1377-91 CrossRef Medline

7. Rodriguez FJ, Scheithauer BW, Port JD. Unusual malignant glioneuronal tumors of the cerebrum of adults: a clinicopathologic study of three cases. Acta Neuropathol 2006;112:727-37 CrossRef Medline

8. Porto L, Jurcoane A, Schwabe D, et al. Differentiation between high and low grade tumours in paediatric patients by using apparent diffusion coefficients. Eur J Paediatr Neurol 2013;17:302-07 CrossRef Medline

9. Kralik SF, Taha A, Kamer AP, et al. Diffusion imaging for tumor grading of supratentorial brain tumors in the first year of life. AJNR Am J Neuroradiol 2014;35:815-23 CrossRef Medline 